\title{
Spontaneous Rupture of Spleen in P.Vivax: A Rare Case Entity
}

\author{
Dr. Anshul Jain ${ }^{1}$,Dr. P.B Gupta ${ }^{2}$ \\ ${ }^{1}$ Resident doctor, Department of Emergency Medicine \\ ${ }^{2}$ Professor \& Head, Department of Emergency Medicine \\ Government Medical College, Surat
}

\section{Introduction}

Malaria has been among the most common infectious diseases in India. The causative microorganism belongs to the Plasmodiumfamily. The four common species that are encountered are Plasmodium falciparum, P. vivax, P. malariaeand P. ovale. Plasmodium falciparuminfection carries more risk of complications such as cerebral malaria, acute renal failure, liver damage, collapse etc.Plasmodium vivaxis considered to be benign but spleniccomplications are more common with P. vivaxinfections ${ }^{2}$. Spontaneous rupture of malarial spleen is uncommon even in the endemic regions of malaria. This may lead to delayed or missed diagnosis of splenicrupture which may be life threatening ${ }^{3}$. Very few case reports are available in literature documenting spontaneous rupture of malarial spleen ${ }^{3}$. We are reporting one such instance of malarial splenicrupture encountered in our department.

\section{Case Report}

We are reporting a case of a patient with P.vivaxmalaria who developed a spontaneous splenic rupture in our department. A40 years old male patient with history of fever with chills since 3 days and abdominal pain since 3 days. There was no history of any associated trauma. On per abdomen examination the only positive finding was of tenderness at the left hypochondrium region.Investigations revealed P.Vivax malaria with Hemoperitoneum on ultrasonography.Patient's CBC report showed HB $5.2 \mathrm{gm} \%$, parasites-rings \&schizontsof $\mathrm{P}$ vivaxseen, platelet count-90000/cumm.Chest X-ray showing pleural effusion on the left side, so pleural fluid tapping was done,tappedfluid was hemorrhagic.Sonography of abdomen revealed a splenic hematoma and an enlarged spleen and liver with free fluid in abdomen. Pelvic-abdominal CT showed a large perisplenichematoma with multiple spleniclacerations.Patient was kept conservatively on fluid therapy as patient was hemodynamicallystable and given necessary anti malarials, antibioticsand blood transfusions.Following this treatment repeat USG abdomen was done after 7 days which showed relatively mild amount of haemoperitoneum. 

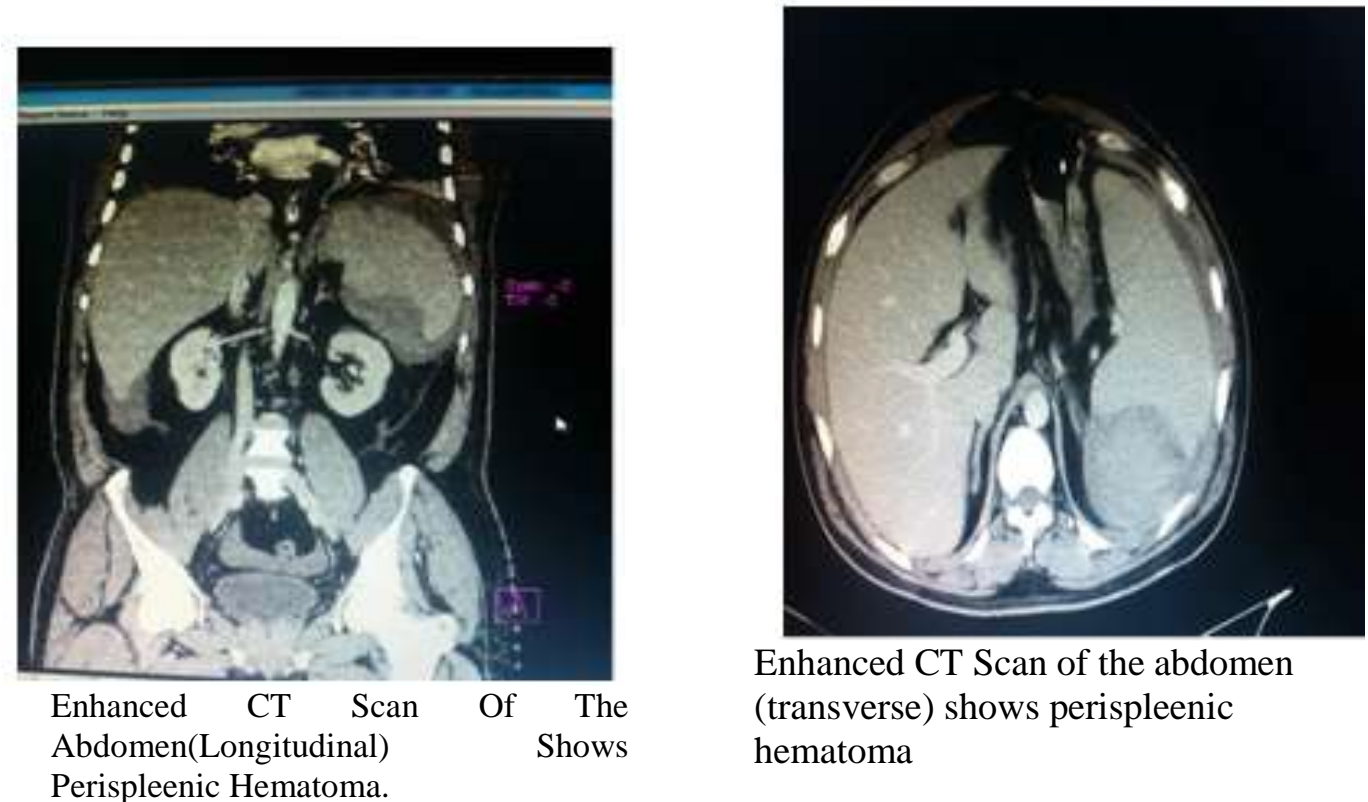

Enhanced CT Scan of the abdomen (transverse) shows perispleenic hematoma

\section{Disscusion}

The causes include infectious, neoplastic, and hematologicaldiseases. Only an estimated $2 \%$ of falciparummalaria cases present with spontaneous splenic rupture ${ }^{1}$. The first case of spontaneous splenic rupture was reported by Atkinson, an English surgeon in $1874^{5}$. A peculiar aspect of this complication is that it can occur in patients taking antimalarial prophylaxis and treatment ${ }^{6}$.Although the exact mechanism of splenicrupture in malaria is still not clear, the following mechanisms have been suggested ${ }^{7,8}$ (i) cellular hyperplasia and congestion leading to increase in intrasplenictension; (ii) spleniccompression by increased intra-abdominal pressure during activities like sneezing, coughing and defecation; and (iii)reticuloendothelialhyperplasia resulting in venous congestion, thrombosis, and infarction,whichcauses sub-capsular hemorrhageand eventual stripping of the spleniccapsule.Clinically, left hypochondrialpain occurring during or following treatment of malaria is the commonest presentation of splenicrupture in malaria ${ }^{8}$. Our case presented with left hypochondrialpain without any history of trauma.

\section{Conclusion}

Spontaneous splenicrupture in complicated vivaxmalaria is extremely rare. Splenicrupture with hemoperitoneumshould be managed with laparotomyand splenectomy, along with antimalarialdrugs. But in this case patient was managed conservatively.Ahigh index of suspicion is needed to detect these complications early.

[1]. Choudhury J, Uttam KG, Mukhopadhyay M:

\section{References}

Spontaneous rupture of malarial spleen. Indian Pediatric, 2008; 45: 327-28

[2]. Strickland GT: Malaria. In: Strickland, G.T

(Ed.), Hunter's Tropical Medicine, seventh ed.

Saunders, Philadelphia, PA, 1991; 586-617

[3]. World Health Organization Action

Programme, 1986. Severe and complicated

malaria. Trans R Soc TropMed Hyg, 1986;

(Suppl): $1-50$

[4]. Wyler DJ: Plasmodium series(malaria). In: MandellGL, Douglas RG Jr., BennetJE (eds.), Principles and Practice of Infectious Diseases, 3rd ed. Churchill Livingstone, New York, 1990; 2056-66

[5]. Atkinson E: Death from idiopathic rupture of the spleen. BMJ, 1874; 2: 403e-404

[6]. VidyashankarC, BasuA, KulkarniAR, ChoudhuryRK: Spontaneous rupture of spleen in falciparummalaria. Indian J Gastroenterol, 2003; 22: 101e102

[7]. Patel MI: Spontaneous rupture of a malarial spleen. Med J Aust, 1993; 159: 836e837

[8]. ZingmanBS, VinerBL: Spleniccomplication in malaria: case report and review. ClinInfect Dis, 1993; 16: 223e232 